\title{
HISTORIA
}

\section{EVOLUCIÓN DE LOS CUIDADOS ENFERMEROS. ANÁLISIS ICONOGRAFICO DESDE LA PERSPECTIVA DE VIRGINIA HENDERSON}

\author{
Mercedes Núñez del Castillo*, Jose Siles González**
}

*Enfermera asistencial del Hospital Universitario de San Juan de Alicante, Antropóloga, Profesora asociada del Departamento de Enfermería de la Universidad de Alicante.

**Catedrático del Departamento de Enfermería de la Universidad de Alicante

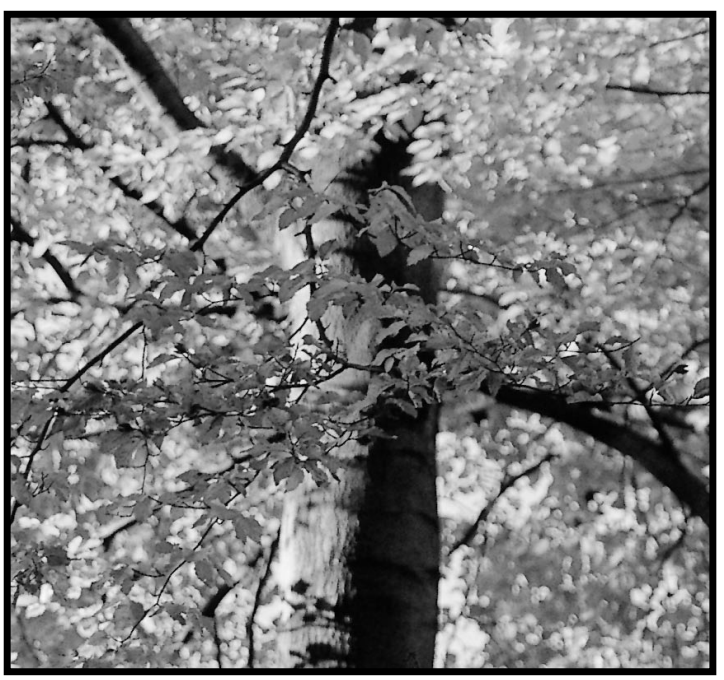

EVOLUTION OF NURSING CARE. AN ICONOGRAPHIC ANALYSIS FROM VIRGINIA HENDERSON'S PERSPECTIVE

\section{ABSTRACT}

The aim of this study is to demonstrate that iconographic material constitutes a source for nursing history. For that purpose, a descriptive, retrospective, comparative, ethnographic historical study was carried out. Iconographic materials come from all human ages and have been classified in categories such as: painting, sculpture, philately and photography. In order to potentiate the hermeneutic process from nursing perspective, categories from Virginia Henderson's needs system have been used. The main conclusion of this study consists in demonstrating pertinence of iconography materials in order to develop historical knowledge of Nursing.

Key words:

history of Nursing, nursing care, anthropology of care, iconography

\section{RESUMEN}

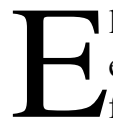

1 objetivo de este estudio es demostrar que el material iconográfico constituye una fuente para la historia de la enfermería. Se ha pretendido alcanzar dicho fin mediante un estudio descriptivo, retrospectivo, comparativo, histórico etnográfico. El material iconográfico utilizado se corresponde con el producido en todas y cada una de las épocas de la humanidad y se ha organizado en categorías como son: pintura, escultura, filatelia y fotografía. Para potenciar el proceso hermenéutico desde la perspectiva de enfermería se ha empleado la categorización del sistema de necesidades de Virginia Henderson. La conclusión principal de este estudio consiste en demostrar la pertinencia del material iconográfico para desarrollar el conocimiento histórico de la enfermería.

\section{Palabras clave:}

Historia de la enfermería, cuidados enfermeros, antropología de cuidados, iconografía. 


\section{INTRODUCCIÓN}

Este trabajo se ha desarrollado mediante un estudio descriptivo, retrospectivo, comparativo, histórico-etnográfico de los cuidados de enfermería, analizado desde la perspectiva de las necesidades básicas de Virginia Henderson, utilizando como fuente la iconografía.

\section{Justificación.}

Alberdi,1986, define la enfermería como:". una disciplina tan antigua como el propio hombre, dado que tiene que ver con todos aquellos elementos y factores materiales y humanos que convergen en una actividad innata y esencial en el ser humano. El cuidado del individuo, el grupo y la comunidad.."(Siles, 1999).

Henderson, en su definición sobre Enfermería hace hincapié en el cuidado, tanto de los individuos enfermos como sanos, la independencia del paciente es un criterio importante para la salud, hasta el punto que en sus escritos equipara salud con independencia,

M.F. Collier, estudiosa de la materia dice que "los cuidados son un conjunto de actos de vida, habituales y cotidianos, que tienen por objeto permitir que esta continúe y se desarrolle". En los orígenes,".. las "prácticas cuidadoras"

ligadas a la conservación de la especie humana.."(Hernández ,1999), o, dar cuidados o cuidar "..era una noción ligada a cualquier forma de vida, y proporcionar cuidados puede considerarse inseparable de la noción de supervivencia de los seres humanos, de la continuidad de la vida en grupo, del desarrollo y mantenimiento de la vida..”.

"..que cuidar representa un conjunto de actos cuya finalidad es la conservar la vida, asegurando la satisfacción de un conjunto de necesidades indispensables para la vida, manifestándose de diferentes formas según los grupos..”(Domínguez, 1986).

Por otro lado, "..los cuidados enfermeros constituyen una constante histórica, que, desde el principio de la humanidad, surge con carácter propio e independiente ..” (Hernández ,1999).

La necesidad de Henderson de comunicarse con otros para expresar emociones, necesidades, temores u opiniones, constituyen las bases esenciales de la relación de ayuda .(Rogers,1986) entre las definiciones que da a la relación de ayuda, dice:"..es aquella en la que uno de los participantes intenta hacer surgir, de una o ambas partes, una mejor apreciación y expresión de los recursos latentes del individuo, y un uso más funcional de éstos.."(Bermejo, 1996), a pesar de que puede adaptarse de forma privilegiada a los cuidados de enfermería, el modelo original, no siempre puede transferirse íntegramente a los cuidados de enfermería , sobre todo en el caso de la entrevista estructurada.

Y según Riopelle, la define como un intercambio ".. siendo a la vez verbal y no verbal, que permite crear un clima de confianza, de respeto y de amor que el cliente precisa para satisfacer sus necesidades fundamentales.."(Phaneuf, 1982).

El hombre primitivo utilizó remedios porque conoció las enfermedades, aunque sigue planteándose una cuestión ¿pero cual era su concepto de enfermedad, como interpretar las situaciones enfermedad o malestar?

Albarracin, opina que pueden existir tres diferentes interpretaciones de enfermedad o malestar (Albarracin, 1987): La enfermedad entendida como penetración en el cuerpo de un objeto extraño, entendida como pérdida o evasión del alma, o entendida como consecuencia inmediata de la acción de los espíritus hostiles.

Para ver los cuidados a lo largo de la historia entre otras fuentes, podemos observar, pintura, escultura o cualquier otra fuente histórica que se nos presente,

Estas fuentes iconográficas reflejan y transmiten la sociedad y la cultura de ese momento, aportando datos importantes sobre el pasado de la actividad cuidadora. “..En consecuencia, cabe afirmar que, desde los orígenes del hombre, las actividades de enfermería tienen su reflejo en los cuidados más elementales, que son los relacionados con la supervivencia: Los cuidados durante el parto, la crianza, la lactancia y el destete.." (Siles, 1999).

Santo Tomás, opina que “..investigar en historia, implica saber utilizar los instrumentos que el tiempo ha ido poniendo a nuestra disposición.." ,...aprender a interpretar los testimonios que otros hombres y mujeres de épocas pasadas nos han dejado como legado..", sigue diciendo esta autora 
más adelante "..existen además otro tipo de fuentes que son manifestaciones artísticas.....estas fuentes llamadas iconográficas..”. “..Es por tanto la iconografía una fuente importante que podemos y debemos utilizar las enfermeras que deseamos conocer nuestro pasado.." (Santo Tomas,1997).

\section{Objetivos.}

El objetivo principal que se plantea es demostrar que el material iconográfico constituye una fuente para la historia de la enfermería.

Y los objetivos específicos son:. Demostrar a través de la iconografía que la profesión enfermera en sus diferentes denominaciones y funciones ha estado presente a lo largo de la historia. Describir y analizar los cuidados de enfermería desde la perspectiva de V. Henderson en la iconografía. Demostrar que los conocimientos empíricos e intuitivos adquiridos por las cuidadoras de forma oral, se transmiten a través de la iconografía. Describir y analizar la relación de ayuda a lo largo de la historia mediante el análisis iconográfico. Demostrar mediante análisis iconográfico, que la iconografía refleja de forma holística las realidades de los cuidados de la salud

\section{Hipótesis nuclear.}

La iconografía constituye una fuente de estudios para la historia de la enfermería.

\section{Otros puntos de partida.}

La profesión enfermera en sus diferentes denominaciones y funciones, ha estado presente a lo largo de la historia en las diferentes fuentes iconográficas

La iconografía constituye una fuente para analizar los cuidados de enfermería desde la perspectiva de Virginia Henderson. Los conocimientos empíricos e intuitivos adquiridos por las cuidadoras de forma oral están presentes en la iconografía. La relación de ayuda, esta presente a lo largo de la historia en las fuentes iconográficas. La iconografía refleja de forma holística las realidades de los cuidados de la salud.

\section{Estado de la cuestión.}

Se han analizado artículos que relacionan la enfermería y la iconografía como instrumento para la investigación, utilizando dos tipos de referencias, los metodológicos y los estudios iconográficos, en los primeros, Santo Tomás $(1996,1997)$, argumenta la importancia de la iconografía para el estudio de la historia de la Enfermería y propone una metodología para el estudio de las iconografías, y, que a su vez es la propuesta por Panofsky, 1995.

Siles, (1999) y Hernández (1999), argumentan un tipo de clasificación y análisis iconográfico muy similar a los autores anteriores, pero dando un toque más acorde a la Enfermería.

Encontramos en la bibliografía análisis iconográficos tanto enfermeros como médicos.

En los estudios iconográficos enfermeros, de pintura se han identificado artículos, de Calvo y Santo Tomas, $(1997,1998)$,Morales y Morente, (2001), García, (1996-97) hace un análisis a través de dos tipos de fuentes: la escrita y la iconográfica. Aunque el autor hace referencia a la fuente iconográfica, de hecho incluye imágenes, no realiza ningún tipo de análisis iconográfico,

En cuanto a fotografía, Lasarte, (2000) y Monge (2001), en sus artículos intentan reconstruir la historia de la enfermería a través de fotografías de la época.

En escultura y vidrieras, Santo Tomás, es la única autora referenciada.

Respecto a los "carteles", como iconografía son "posters" relacionados con enfermería y con salud; donde se reflejan las comidas para diabéticos, incidencias de úlceras por presión.

Guerra (2001), Ballesteros (2002) y Miralles (1995, 1999), pretenden conocer y aportar como la enfermería y los cuidados han estado presentes a través de la filatelia, haciendo un recorrido y descripción de los sellos publicados tanto a nivel nacional como a nivel internacional

En cuanto a los análisis iconográficos médicos, González, (1996), Moran, (1996), Grau, (2001), Castillo (2002), llegan a un diagnóstico médico mediante el análisis de las la iconografías. Del Águila, (2003), analiza y explica las costumbres de la época, relacionadas con la salud y la enfermedad.

Soriano, /Fotomedic, (desde el 2000), han proporcionado fotografías de enfermeras y de cuidados, aunque lo extrapolan al enfermo, la ayuda, la soledad, las patologías o los lugares del cuidado. 


\section{METODOLOGÍA.}

El material iconográfico que se va a utilizar para verificar o refutar las hipótesis es tanto civil como religioso, obtenido mediante búsqueda iconográfica, analizándolo desde principio de la historia hasta nuestros días, y organizados por categorías como son: pintura, escultura, filatelia y fotografía.

Las variables, también están distribuidas por categorías y corresponden a las 14 necesidades básicas de Henderson, siendo estas: 1. Respirar, 2. Beber y comer, 3. Eliminar, 4. Moverse y mantener una buena postura, 5. Dormir y descansar, 6 . Vestirse y desvestirse, 7. Mantener la temperatura corporal dentro de los límites normales, 8. Estar limpio, aseado, y proteger sus tegumentos, 9. Evitar los peligros, 10. Comunicarse con sus semejantes, 11. Actuar según sus creencias y valores, 12. Ocuparse para realizarse, 13 recrearse, 14. Aprender haciendo diferenciación entre dependencia o independencia, la relación de ayuda se analiza como categoría independiente.

\section{Análisis de los datos.}

Los datos cualitativos se han recogido a través de la observación de las iconografías seleccionadas y se llevan a cabo dos tipos de análisis, el iconográfico propiamente dicho y el antropológico.

Para las iconografías, se va a utilizar la interpretación iconográfica propuesta por Panofsky “.. y que se realiza en tres momentos, descripción preiconográfica, Análisis iconográfico e Interpretación iconográfica. El método iconológico va a permitir conjuntar el conocimiento de la historia, las tradiciones, costumbres y cuidados enfermeros. (Santo Tomas, 1997).

Para realizar los análisis iconográficos de pintura y escultura se va a utilizar los esquemas propuestos por Siles,(1999) .

Para la interpretación iconográfica de los cuidados de enfermería, se va a utilizar el análisis propuesto por Henderson (Riopelle) para el estudio de las 14 necesidades.

Para realizar el análisis cuantitativo se ha utilizado el programa Excel del Windows XP.

Para realizar el análisis cualitativo se ha utilizado Heurística y Hermenéutica

La búsqueda HEURÍSTICA se realiza mediante la observación documental y mediante fuentes indirectas constituidas por documentos e iconografías, en cuanto al Análisis cualitativo nos apoyamos en la HERMENÉUTICA Y FENOMENOLOGÍA

Para estructurar la investigación, se va a clasificar la Historia, según el esquema temporal propuesto por Siles, Hernández, etc., y que tiene una correspondencia con los propuestos por otros expertos en historia del arte.

Para mejorar la operatividad en el estudio de historia de la enfermería se acepta un modo de clasificar esta historia en tiempos convencionales, el esquema temporal es el propuesto por Childe y Siles.

\section{Marco teórico.}

El objetivo de Henderson fue, expresar de forma clara y concisa la función propia de la enfermería, teniendo presente las influencias tanto positivas como negativas. Dedicó la mayor parte de su vida a formarse y a investigar, creando e impulsando el concepto de enfermería tal como la conocemos hoy, fue la pionera en la incorporación de una filosofía humanista y existencialista a los cuidados enfermeros.

De hecho, Henderson considera su trabajo más como una aserción filosófica, como una definición, que como una teoría, ya que el término teoría no se utilizaba en el momento en que ella formuló sus ideas. Desarrolla sus ideas motivada por sus preocupaciones sobre la función de la enfermera y su situación jurídica. (Wesley, 1997). En su libro La naturaleza de la Enfermería, publicado en 1966 ofrece una definición concisa de la Enfermería en la que destaca la concepción del receptor de los cuidados como agente de cuidados de salud, otorgando a la enfermera un rol complementario/suplementario en la satisfacción de las 14 necesidades básicas definidas en su modelo. (Fernández, 1994).

En 1955, la American Nurses Association, aprobó la definición de la práctica de enfermería propuesta por su comité de legislación. Esta sigue siendo la versión original publicada de nuevo en 1962. Opina Henderson: “..que aunque aún muy general, sugiere al menos que la enfermera puede observar, cuidar y consolar al paciente y supervisar a otros miembros del personal sanitario sin ser ella supervisada por el médico..". 
Pero no fue hasta 1940 , que pudo probar sus ideas en la práctica real, desarrollándose el único estudio avanzado en la enfermería médico-quirúrgica. “..Este curso era único porque hablaba del cuidado individualizado del paciente y estaba organizado alrededor de los problemas principales de la enfermería en vez de en los diagnósticos médicos y en las enfermedades somáticas.." (Henderson, 1994).

En 1958, el Nursing Service Comité of the Internatinal Council of Nurses le pide que preparara un boletín de enfermería básica, el boletín publicado en 1961, se cita: “..La función singular de la enfermería es asistir al individuo, enfermo o no, en la realización de esas actividades que contribuyen a su salud o su recuperación (o a una muerte placentera) y que el llevaría a cabo sin ayuda si tuviera la fuerza, la voluntad o el conocimiento necesario. Y hacer esto de tal manera que le ayude a adquirir independencia lo más rápidamente posible..”.

“..Creo que la enfermera es, y debe ser legalmente independiente capaz de hacer juicios independientes mientras no haga diagnósticos, prescriba tratamientos o emita pronósticos, porque son funciones del médico. Pero la enfermera es la mayor autoridad en los cuidados básicos de enfermería. (Henderson, 94).

En 1966, perfiló su definición de enfermería, desarrolló su definición basándose en las ciencias de la fisiología, medicina, psicología y física. Haciendo hincapié en el cuidado tanto de los individuos enfermos como sanos, incorporando los aspectos espirituales al cuidado enfermero. (Wesley, 1997). Virginia Henderson no cita directamente los cuatro conceptos del paradigma enfermero, por lo que son adaptados a partir de publicaciones. (Marriner, 2003, Wesley,1997)..

Henderson define la ENFERMERÍA como la asistencia fundamentalmente al individuo enfermo o sano para que lleve a cabo actividades que contribuyan a la salud, a una muerte tranquila;. Requiere trabajar de forma interdependiente con otros miembros del equipo de salud, ayuda al paciente a satisfacer las 14 necesidades básicas, realiza un cuidado individualizado y utiliza un plan de cuidado enfermero por escrito. (Wesley, 1997).

PERSONA “..Es considerada por Henderson como un individuo que necesita asistencia para alcanzar un estado de salud e independencia o para morir con tranquilidad, la familia y la persona son consideradas como una unidad..”, “..consta de componentes biológicos, psicológicos, sociales y espirituales..”, y “.. se esfuerza por obtener un estado de independencia..”(Wesley, 1997), las personas “.. tienen necesidades básicas reales de supervivencia..”.

SALUD. “.. se refiere a la capacidad del individuo para funcionar con independencia en relación a las 14 necesidades básicas ..”, “... requiere fuerza, voluntad o conocimiento..” (Wesley, 1997).

ENTORNO. Henderson no lo define explícitamente. ".. los individuos sanos son capaces de controlar el entorno, pero la enfermedad puede alterar esta capacidad..”, “..las enfermeras deben proteger a los pacientes de las lesiones mecánicas.... (Marriner, 2003).

Henderson identifica 14 necesidades básicas que son los componentes del cuidado enfermero. Por tanto considerando estas 14 necesidades básicas de forma conjunta proporcionan un enfoque holístico de la enfermería. (Wesley, 1997).

\section{Búsqueda bibliográfica.}

Se ha realizado la búsqueda bibliográfica en las bases de datos de enfermería CUIDEN, CUIDATGE, BDI, ENFISPO, MEDLINE, utilizando en primer término las palabras clave, enfermera, enfermería, cuidados de enfermería, arte, iconografía, pintura, escultura, fotografía, carteles y filatelia, tanto en español como en inglés para realizar la búsqueda en MEDLINE. Dado que el número de artículos no era suficiente, se realiza una segunda búsqueda añadiendo los términos enfermedad, salud, patología y medicina, así mismo en español como en inglés. Como sigue siendo limitado el número de referencias encontradas, se procede al vaciado de algunas revistas que están relacionadas pero no indexadas en las bases de enfermería.

El descriptor "arte", no ha resultado ser positivo, ya que el término es entendido como "arte en el cuidar", "arte de la enfermería",etc.; tampoco es muy útil la variable "carteles", donde se reflejan las comidas para diabéticos, incidencias de úlceras por presión, el resto son bases para concursos de carteles. 


\section{RESULTADOS}

De las 103 iconografías analizadas, se observan claramente 298 necesidades de las descritas por Virginia Henderson, de estas un $49 \%$ son independientes y el resto, $51 \%$ dependientes, (gráfico 1 ).

En la prehistoria, todas las necesidades observadas son de independencia, y siendo la de vestir, actuar según sus creencias y valores y la de aprendizaje las más representadas. (Gráfico 2).

En la edad antigua, se mantiene un equilibrio entre las manifestaciones de dependencia (42\%) y las de independencia (58\%), centrándose en las necesidades fisiológicas o de supervivencia y en las de afecto y autorrealización, apareciendo por primera vez la relación de ayuda en una iconografía. (Gráfico 3)

En la Edad Media, los porcentajes de representación de las necesidades son un $57 \%$ para las de independencia, siendo las necesidades de moverse y mantener una buena postura y la de dormir y descansar las más representadas, en un 18 y un $19 \%$ respectivamente, el resto de las necesidades están muy dispersas. La relación de ayuda sigue en dos de las iconografías analizadas. (Gráfico 4)

En la edad Moderna, Los porcentajes de representación de las manifestaciones de dependencia/ independencia, representan un $57 \%$ para las necesidades de dependencia y un $43 \%$ para las de independencia, siendo la necesidad de moverse y mantener una buena postura y la de dormir y descansar las más representadas. Suponiendo un $26 \%$. La relación de ayuda vuelve a aparecer en una de las iconografías analizadas. (Gráfico 5)

En la edad contemporánea, el 36\% de las necesidades representadas corresponden a manifestaciones de independencia, mientras que el $64 \%$ corresponden a las de dependencia, siendo las necesidades de moverse y mantener una buena postura $(8 \%)$ y la de dormir y descansar $(31 \%)$ las más representadas. (Gráfico 6)

Los resultados analizados por necesidades de Henderson (Tabla 1), son los siguientes:

DORMIR Y DESCANSAR. Es la necesidad más representada en las iconografías analizadas, tanto mediante manifestaciones de dependencia como de independencia, MANTENER UNA BUENA POSTURA. Es la segunda necesidad más representada, siendo el $64 \%$ manifestaciones de dependencia. La necesidad de BEBER Y COMER. Es la tercera, está representada en todas las etapas de la historia, representa un $0,8 \%$, siendo el $75 \%$ de estas manifestaciones de dependencia. ESTAR LIMPIO, ASEADO Y PROTEGER LOS TEGUMENTOS y EVITAR LOS PELIGROS.

Necesidades que aparecen representadas a partir de la Edad media, en forma de manifestación de dependencia en un $67 \%$ y $69 \%$ de los casos, aunque es escasa su representación $(0,04 \%)$,

La necesidad de COMUNICACIÓN. Y ACTUAR SEGÚN CREENCIAS, representan un 6,37\%. APRENDER, VESTIRSE Y DESVESTIRSE y OCUPARSE PARA RECREARSE. son menos frecuentes, MANTENER LA TEMPERATURA DENTRO DE LOS LÍMITES NORMALES. Casi invisible en las iconografías analizadas, solo en 3 ocasiones se representa esta necesidad, en la Edad Media y en la Moderna, la necesidad de RESPIRAR, es la menos observada en las iconografías analizadas, solo aparece en la edad moderna y contemporánea y siempre en su aspecto de dependencia. (gráfico).

\section{DISCUSIÓN.}

En la Prehistoria todas las necesidades observadas son en forma de manifestación de independencia, las necesidades se manifiestan mediante valores indirectos o instrumentos.

El hombre adulto tiene conocimiento de su entorno y por tanto toma medidas, siendo este uno de los factores biológicos que van a influir en la satisfacción de la necesidad de evitar los peligros, aunque curiosamente esta necesidad solo aparece representada en una ocasión.

El aprendizaje como valor sociológico, no es precisamente una necesidad ampliamente observada de forma objetiva, pero si de forma implícita.

En la edad antigua, la dependencia de las necesidades analizadas, son como consecuencia de actividades o de manifestaciones representadas en las iconografías.

En la edad moderna y contemporánea, se observa la dependencia de las necesidades en las tareas de las cuidadoras, y mediante signos indirectos observados en las iconografías.

En la necesidad de comunicación de Henderson, a la sexualidad le falta especificidad. 
La relación de ayuda, empieza a observarse a partir de la edad Antigua, aunque en una proporción mínima, se mantiene constante hasta nuestros días

$\mathrm{Al}$ analizar los capiteles, metopas y canecillos no se puede realizar de forma individual exclusivamente pues constituyen en la mayoría de las ocasiones una continuidad dentro del monumento.

Se ha constatado la falta de especificidad de algunas palabras clave al realizar la búsqueda bibliográfica, pues son entendidas de forma diferente a los intereses del estudio.

\section{CONCLUSIÓNES.}

Con la muestra analizada han alcanzado los objetivos y se confirman las hipótesis que se plantean, llevando a las siguientes conclusiones:.

Que La iconografía representa una fuente para estudiar los cuidados de enfermería y la historia de la profesión.

Que la profesión enfermera, en sus diferentes denominaciones y funciones ha estado presente en las iconografías analizadas a lo largo de la historia, desde la cuidadora intuitiva dedicada a los cuidados de supervivencia, pasando por las órdenes religiosas dedicadas al cuidado hasta los profesionales actuales. Siendo la edad contemporánea la que facilita más información, concretamente la fotografía.

La iconografía es una fuente para analizar los cuidados desde la perspectiva de Virginia Henderson, siendo las pinturas rupestres las primeras manifestaciones donde se observan estas necesidades.

Los conocimientos empíricos e intuitivos que han adquirido las cuidadoras de forma oral están presentes en las iconografías.

La relación de ayuda empieza a estar presente a partir de la Edad Antigua, observándose iconografías donde se representa exclusivamente la comunicación terapéutica e iconografías donde la relación de ayuda está presente junto a otras necesidades de Henderson.

Y se ha demostrado mediante el análisis iconográfico, que las diferentes formas de iconografía reflejan de forma holística las realidades de los cuidados enfermeros.

En España apenas hay constancia de artículos que relacionen el tema de la Enfermería y los cuidados con la iconografía, referenciándose los primeros a partir de 1996.

La mayoría de los trabajos se ciñen a la interpretación iconográfica directa.

Los períodos de estudio referenciados comienzan en la Edad Media, siendo la iconografía elegida para el estudio de la enfermería y sus cuidados la pintura principalmente.

A pesar que la necesidad de aprendizaje no está muy presente en las iconografías a lo largo de la historia, éstas suponen un medio directo de aprendizaje.

El estudio aporta una base para realizar en el futuro trabajos comparativos con otras culturas.

\section{BIBLIOGRAFÍA.}

ALBARRACIN, A.(1987): El remedio terapéutico en el mundo primitivo 1:16-36 en Historia del medicamento. Editorial Doyma S.A.

BALLESTEROS ALVARO, A. M. (2002): La enfermería y los cuidados en los sellos de España. Cultura de los cuidados: revista de enfermería y humanidades. VI $n^{\circ} 11: 24-27$.

BERMEJO,J.C, (1996). Apuntes de relación de ayuda. Cuadernos del Centro de Humanización de la Salud.

CALVO, M. Á. (2001): Análisis iconográfico de la pintura de Lucas Valdés 'Asistencia a los sacerdotes en la enfermería. Publicación: Alcalá de Guadaira, Sevilla Pp. 61.

CIBANAL JUAN, L, (et al). (2003): Técnicas de comunicación y relación de ayuda en ciencias de la salud. Editorial Elsevier España.

COLLIÈRE, M. (1989). Utilización de la antropología para abordar las situaciones de cuidados. Rol, 179$180,17-25$.

DOMINGUEZ-ALCÓN, C. (1986): Los cuidados y la profesión enfermera en España.Editorial Pirámide.

GUERRA GONZÁLEZ, JE. (2001): Historia de la enfermería. Reflejo en la filatelia mundial. Alcalá de Guadaira, Sevilla: Qalat Chábir , pp. 61-62

GONZÁLEZ IGLESIAS, J. (1996): Patografía bucal, sacamuelas y dientes de ahorcados en la pintura de Francisco de Goya. Noticias médicas. N 3622, junio (35-38).

GRAU, JUAN J; (et al). (2001): Cáncer de mama en los cuadros de Rubens y Rembrandt. Medicina Clínica Marzo 116 (10):380-384.

HERNÁNDEZ CONESA, J. (1999): Historia de la Enfermería. Un análisis histórico de los cuidados de Enfermería. Mc. Graw-Hill Interamericana. 
HENDERSON, V. A. (1994): La naturaleza de la enfermería. Una definición y sus repercusiones en la práctica, la investigación y la educación. Reflexiones 25 años después.. Mc Graw-Hill Interamericana de España S.A.

LASARTE CALDERAY, J. E. (2000): Fuentes iconográficas en la revista Nuevo mundo (1896-1930). Híades: Revista de Historia de la Enfermería; 7: 11-54

MARRIMER TOMEY, A Y MARTHA RAILE ALLIGOOD. (2003): Modelos y teorías en enfermería. Elsevier España S.A. Madrid.

MAYAN CENDÓN, M D, (et al) :La historia de la Escuela de Enfermeras de Santa madrona a través de la fotografía: 1917-1936. Alcalá de Guadaira. Sevilla. Pp. 63-64. .

MIRALLES SANGRO, MT (et al). (1995): San Juan de Dios y su Orden en la filatelia. Index Enfermería (14) pp.59-61.

(1999): La iconografía enfermera en la filatelia. Sevilla. Asociación de Enfermería Docente. Pp 585-592.

MONGE JUAREZ, M. (2001): Enfermeras y enfermos a través de sus fotografías, Lima 1914.Hiades. Año VI (8): 599-600.

MORALES MONTALVA, M .S. (et al). (2001): La lactancia materna a través del arte de las Cantigas de Santa María de Alfonso X El Sabio. Híades: Año VI (8): 561-562.

MORAN SUÁREZ, I. (1996): El fuego de San Antonio: Estudio del ergotismo en la pintura del Bosco. Asclepio. Revista de historia de la medicina y de la ciencia. Vol: XLVIII -2 - (173-193).

MORENTE PARRA, M. I. (2001): La Virgen como cuidadora y sanadora a través de las Cantigas de Santa María de Alfonso X .Híades: Año VI(8):337-341.

PANOFSKY,E (1995): El significado en las artes visuales. Alianza Editorial.

PHANEUF, M (1982):La comunication et la relation d'aide: éléments de compétence de l'infirmière, $2^{\mathrm{a}}$ parte, Nursing Quebec, Vol $2: 3$ marzo, abril.
PUJOL CARMONA, C (et al). (2001): Enfermería y arte: apunte iconográfico de los cuidados a través de la historia. Alcalá de Guadaira, Sevilla. Pp. 76

RIOPELLE,L. (1993): Cuidados de enfermería. Un proceso centrado en las necesidades de la persona. Editorial Interamericana. Mc. Graw-Hill.

ROGERS,CR .(1986): El proceso de convertirse en persona. Paidos, Barcelona.

SANTO TOMÁS PÉREZ, M. (et al). (1996) : Iconografía: un instrumento para la investigación histórica. Index Enferm V(16-17):87-88 .

(1997): "Iconografía y enfermería : un instrumento para la investigación histórica"

Index de Enfermería; 19: 13-16

SANTO TOMÁS PÉREZ, M (et al). (1998): Santa Isabel bañando a los tiñosos: análisis iconográfico. Index Enferm. Año VII (22):47-49.

(1998): Picasso: Ciencia y caridad. Análisis iconográfico. Index Enfermería año VII (23): 39-40. (1999) :Los cuidados vistos a través de la iconografía del Camino de Santiago. Publicación: Gijón. Congreso: IV Congreso Nacional de Historia de la Enfermería. La Enfermería en las Rutas Jacobeas: Perspectiva Histórica. Gijón. 6, 7 y 8 de mayo de 1999: Pp. 16.

SANTO TOMÁS PÉREZ, M. (2001): Iconografía y cuidados de enfermería. Alcalá de Guadaira, Sevilla: Pp. 77-78.

SANTO TOMÁS PÉREZ, M (2003): Historia de la enfermería. En: Enfermería fundamental, serie manuales de enfermería. Directora de la serie: Blasco, Rosa, M. Fernández, C; Garrido, M; Santo Tomás, M; Serrano, D; colaboración Fuentes, R. Masson S.A. Barcelona

SILES GONZÁLEZ, J. 1999: Historia de la Enfermería volumen I y II. Consejo de enfermería de la Comunidad Valenciana.

WESLEY, R, L. (1995): Teorías y modelos de enfermería. $2^{a}$ edición. Ed. Mc Graw-Hill Interamericana México.

\section{Relación dependencia-independencia}

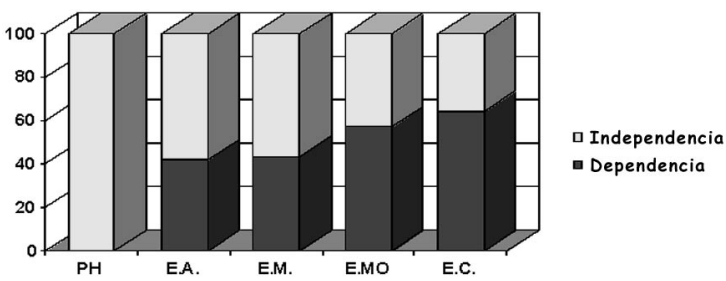

\section{Prehistoria}

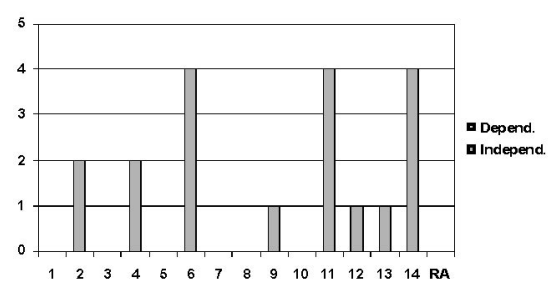

Gráfico 2 
Edad Antigua

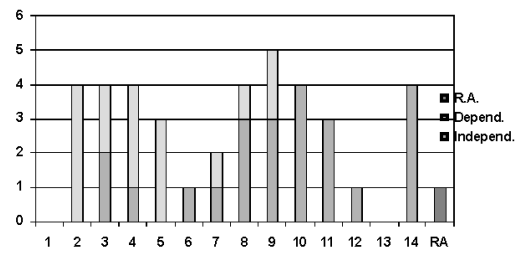

Gráfico 3
Edad Media

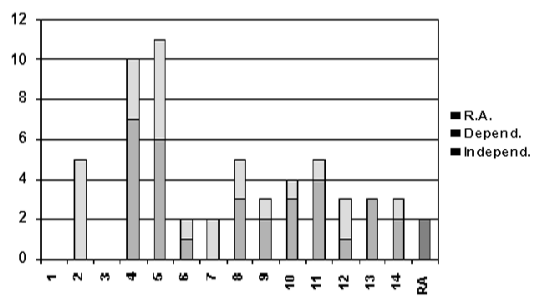

Gráfico 4
Edad Contemporánea

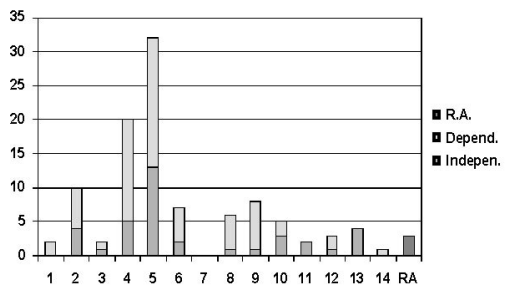

Gráfico 5
Distribución de las necesidades

de Henderson

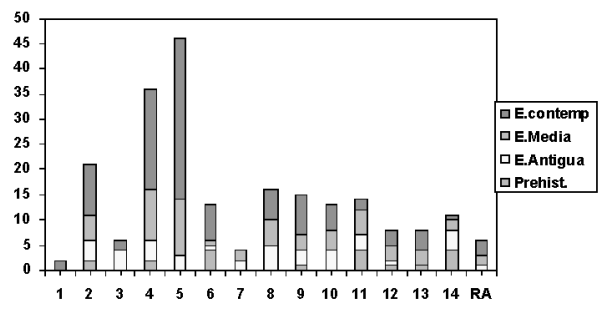

Gráfico 6

TABLA 1

RELACIÓN DE NECESIDADES DE HENDERSON EN EL CONJUNTO DE LAS ICONOGRAFÍAS ANALIZADAS

\section{N. Henderson}

5 Dormir y descansar

4 Moverse y postura ade.

2 Comer y beber

8 Higiene e integridad piel

9 Evitar peligros y lesionar

10 Comunicación

11 Actuar según creencias

14 Aprendizaje

6 Vestirse y desvestirse

13 Recreación

12 Ocuparse para realizarse

3 Eliminación

RA Relación ayuda

1 Respirar

\section{Frecuencia (n)}

66

52

28

22

22

19

19

15

13

10

9

8

7

3

298
Proporción

0.2214

0.1744

0.0939

0.0738

0.0738

0.0637

0.0637

0.0503

0.0436

0.0335

0.0302

0.0268

0.0234

0.0100
Porcentaje

22.14

17.44

9.39

7.38

7.38

6.37

6.37

5.03

4.36

3.35

3.02

2.68

2.34

1.00

100 